NASA Technical Memorandum 101411

ICOMP-88-22

\title{
Three-Dimensional Marginal Separation
}

Peter W. Duck

Institute for Computational Mechanics in Propulsion

Lewis Research Center

Cleveland, Ohio

(AASA-TH-101411) THREE-DIAEESICHAL MARGINAL
SEFABATICA (NASA) $33 \mathrm{~F}$

December 1988

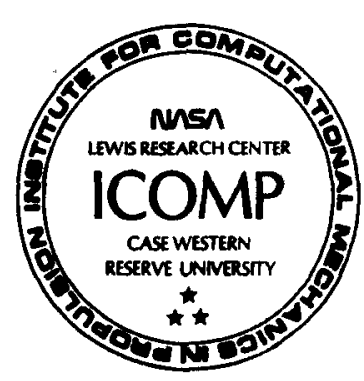


Three-Dimensional Marginal Separation

Peter W. Duck*

Institute for Computational Mechanics in Propulsion

Lewis Research Center

Cleveland, Ohio 44135

\section{SUMMARY}

The three-dimensional marginal separation of a boundary layer along a line of symmetry is considered. The key equation governing the displacement function is derived, and found to be a non-linear integral equation in two space variables. This is solved iteratively using a pseudo-spectral approach, based partly in double Fourier space, and partly in physical space. Qualitatively the results are similar to previously reported two-dimensional results (which are also computed to test the accuracy of the numerical scheme); however quantitatively the three-dimensional results are much different.

*Work funded under Space Act Agreement C99066G; presently at Department of Mathematics, University of Manchester, Manchester, England. 


\section{INTRODUCTION}

High Reynolds number flows may be broadly categorised into two classes, namely attached and detached. The former class generally involves the confinement of a viscous (i.e. boundary) layer to the immediate vicinity of the flow boundary, the flow elsewhere being generally inviscid. A classical example of this is the Blasius flow over a semi-infinite flat plate. The detached class of high Reynolds number flow involves viscous layers occuring in the main body of the fluid (as well as on the flow boundaries). An important example of a flow of this class is that of the steady flow past a circular cylinder. This problem has been investigated numerically (for finite Reynolds numbers) by Fornberg $(1980,1985)$ and models for asymptotically large Reynolds number situations been described by Smith $(1979,1985)$ and by Peregrine $(1985)$, although the precise details of the ultimate high Reynolds number flow in this, (and similar important cases) are still open to speculation. Generally, attached flows may be thought of as being associated with streamlined bodies, whilst detached flows are more likely to be associated with bluff bodies.

One reason for the great theoretical difficulty in describing detached flows is that generally there appears to be no continuous transition from the fully attached to the grossly detached state. A classical example of this is the work of Goldstein (1948), who showed convincingly, for the first time how a boundary layer with an adverse pressure gradient would terminate in a singularity right at the the point of separation. Some years later Stewartson (1970) showed that this singularity could not be removed or alleviated with the inclusion of a triple deck.

The problem studied by Stewartson et al (1982) (hereafter refered to as $I$ ) was that of two-dimensional marginal separation. Here the question was the behaviour of a boundary layer which is on the verge of separating at a point, but then recovers. This work showed how an interaction region was formed, centred at the point of zero wal! shear, which served to smooth - out the discontinuous streamwise gradient of the shear 
stress at this point. Further, it was shown in I (by the solution of a non-linear integral equation) that by means of varying an arbitary parameter

( $\Gamma$ in their notation) that : (i) separated flow was possible within the interaction zone; (ii) for certain values of $\Gamma$ the solution exhibited non-uniqueness; (iii) there exists a critical value of $\Gamma$ above which no solution exists. (This latter conclusion is a further example of a lack of uniform transition from a fully attached to a grossly detached state.) A thorough investigation of the aformentioned integral equation was mounted by Brown and Stewartson (1983), and this revealed that up to four solutions were possible for certain values of the parameter $\Gamma$.

One of the motivations for this work was some numerical investigations of laminar boundary layers on smooth bodies of revolution at incidence, carried out by Cebeci et al (1980). In this study, the classical boundary layer equations along the line of symmetry were integrated numerically, and it was found that at one particular angle of incidence ( $40^{\circ}$ for the case cited), the skin friction dipped (linearly) to zero at one location, and then rose quickly again. At a very slightly increased angle of incidence $\left(41^{\circ}\right)$ the separation process turns out to be catastrophic (although this is not unexpected, due to the non-interacting nature of the calculation). More recently, Cebeci and Su (1988) have considered the full three-dimensional bounclary layer problem on a prolate spheroid, focusing much attention on the separation aspect. Smith (1982) considered the unsteady counterpart of the problem considered by $I$, and found that a finite-time breakdown of the solution was possible, which he attributed to the occurence of dynamic stall.

In an attempt to model the Cebeci at al (1980) configuration rather better, Brown (1985) (hereafter referred to as II) considered the problem of marginal separation of a boundary layer along a line of symmetry. The result of the analysis was again an integral equation, althougb this time two constants were found necessary (although these turn out to be related). One important assumption with this work is an a priori knowledge of the crossflow pressure gradient (obtained from inviscid theory), and so this work mä be described as mildly three- dimensional, although it does have interesting and imporant 
differences from the two-dimensional work of 1 . In particular, in general the upstream streamwise gradient of skin friction is no longer minus the downstream gradient (the ratio of these quantities being a parameter to the problem).

The aim of this paper is to treat a fully three-dimensional marginal separation (again along a line of symmetry), incorporating crossflow pressure variations in the interaction zone. Following $I$ and $I I$ we assume the boundary layer has a particular form at the separation point, which lies on the line of symmetry. Defining the streamwise direction to be parallel to the line of symmetry, and the crossflow direction perpendicular to the line of symmetry, we then make a key assumption (akin to that used in I and II), that the streamwise component of skin friction vanishes simultaneously (at the separation point) with the crossflow derivative of the crossflow component of skin friction. The precise details will be described in the following section.

Work on three-dimensional interacting boundary layers (a category to which this work relates) has progressed somewhat slowly. Smith et al (1977) posed the three-dimensional triple deck problem, which they then solved for a linearised case. Duck and Burggraf (1986) used a pseudo-spectral method to treat the fully non-linear version of Smith el al (1977), and obtained solutions for a number of separated flows. Smith (1983) (extended by Bodonyi and Duck 1988) presented a finite-difference scheme for treating flows of this type, using a system of rotated coordinates, enabling the problem to be made quasi-two-dimensional. The amount of literature on three-dimensional separation remains small, in spite of its great importance; this would appear to be due to the great numerical difficulties encounted with flows of this type. Indeed the author is unaware of any detailed studies of the three-dimensional Goldstein singularity per se (although Cebeci et al 1980 do have some discussion of this point).

The outcome of the analysis of this paper is a two-dimensional non-linear integral equation (or partial-integro-differential equation depending on the final form used). (The work of I and II resulted in an analogous one-dimensional equation.) In order to solve tinis equation, we shall adopt a pseude-spectral approack, with the solution 
technique being based partly in Fourier space, and partly in physical space. This type of approach is in the spirit of Duck and Burggraf (1986), although the details are very much different. It appears that this type of approach has considerable advantages over solution procedures confined to physical variables. 


\section{BASIC FORMULATION}

Let us consider an incompressible fluid of kinematic viscosity $\cdots$ Suppose that $L$ denotes a typical lengthscale in the problem (for example the distance between a leading edge/stagnation point to the separation point), and that a typical freestream velocity directed along the centreline is $U_{\infty}$. Then we take Cartesian coordinates ( $\left.L x, L y, L z\right)$, origin at the separation point; the flow velocity is $U_{\infty} \underline{u}=U_{\infty}(u, v, w)$, and we define the centreline to lie along $z-0$. The body surface (ignoring any curvature terms) lies on the plane $y=0$. The pressure is written as $\rho U_{\infty}{ }^{2} p$, where $\rho$ is the fluid density. The Reynolds number (assumed large throughout this paper) is defined as

$$
R=U_{\infty} L / v \text {. }
$$

The governing non-dimensional equations are then written as

$$
\begin{gathered}
\nabla . \underline{\mathbf{u}}-0 \\
(\underline{\mathrm{u}} \cdot \nabla) \underline{\mathrm{u}}=-\nabla \mathrm{p}+\mathrm{R}^{-1} \nabla^{2} \underline{\mathrm{u}} .
\end{gathered}
$$

We shall suppose that all three velocity components are (generally) non-zero and functions of $x, y, z$. Because of the symmetry of the problem about $z=0$, we expect $u, v$ and $p$ to be even functions about the centreline, whilst will be an odd function of $z$.

Finally, on $y=0$ we require to impose the usual conditions of zero velocity, i.e.

$$
\underline{u}=0 \text { on } \quad y=0 \text {. }
$$




\section{NON-INTERACTING FLON}

As the flow approaches the point of zero skin friction $(x-y=z-0)$ we suppose that the boundary layer has the standard thickness $O\left(R^{-\frac{1}{2}}\right)$. However, rather than allowing the boundary layer at this point to have a Coldstein (1948) "square-root" singularity, we assume that ambient conditions are such that this is avoided. This we achieve (following I and 11 ) by a careful choice of condtions (as defined in (3.11) below), which results in the boundary layer exhibiting a streamwise gradient of streamwise wall shear (and crossflow derivative of streamwise gradient of crossflow wall shear) that is discontinuous at $x=z-0$. We now seek to determine the general form of this solution under these conditions, in this region. Taking (2.2), (2.3) and introducing classical boundary-layer variables,

$$
\begin{aligned}
& y=R^{-\frac{1}{2}} Y \\
& V=R^{-\frac{1}{2}} V
\end{aligned}
$$

with all other variables (both dependent and independent) remaining unchanged, then the three-dimensional boundary layer equations can be written (to leading order)

$$
\begin{aligned}
& \frac{\partial u}{\partial x}+\frac{\partial v}{\partial Y}+\frac{\partial w}{\partial z}=0 \\
& u \frac{\partial u}{\partial x}+v \frac{\partial u}{\partial Y}+\frac{\partial u}{\partial z}=-\frac{\partial p}{\partial x}+\frac{\partial^{2} u}{\partial Y^{2}} \\
& u \frac{\partial w}{\partial x}+v \frac{\partial w}{\partial Y}+v \frac{\partial w}{\partial z}--\frac{\partial p}{\partial z}+\frac{\partial^{2} w}{\partial Y^{2}} \\
& \frac{\partial p}{\partial Y}=0 .
\end{aligned}
$$

We now assume the following solution expansions (in line with our earlict remarks) 


$$
\begin{aligned}
& u-U_{0}(Y)+\mu\left(\lambda^{2} x^{2}+z^{2}\right)^{\frac{1}{2}} U_{0}^{\prime}(Y) \\
& -x\left[w_{1}(Y)+v_{1}^{\prime}(Y)\right]+\frac{1}{2} x^{2}\left[-v_{2}^{\prime}(Y)-w_{2}(Y)\right] \\
& +\frac{1}{2} \mu^{2}\left(\lambda^{2} x^{2}+z^{2}\right) U_{0}^{n}(Y)+z^{2} u_{23}(Y) \\
& -\mu x\left(\lambda^{2} x^{2}+z^{2}\right)^{\frac{1}{2}}\left(V_{1}^{n}+w_{1}^{\prime}\right)+\ldots \\
& V=V_{1}(Y)-\frac{\mu \lambda^{2} x}{\left(\lambda^{2} x^{2}+z^{2}\right)^{\frac{1}{2}}} U_{0}(Y)+x V_{2}(Y) \\
& -\mu^{2} \lambda^{2} \times U_{0}^{\prime}(Y)-\mu\left(\lambda^{2} x^{2}+z^{2}\right)\left(w_{1}(Y)-V_{1}^{\prime}(Y)\right) \\
& +\frac{x^{2} \lambda^{2} \mu}{\left(\lambda^{2} x^{2}+z^{2}\right)^{\frac{1}{2}}}\left(2 w_{1}+v_{1}^{\prime}\right)+\ldots \\
& w=z w_{1}(Y)+x z w_{2}(Y)+\mu z\left(\lambda^{2} x^{2}+z^{2}\right)^{\frac{1}{2}} w_{1}^{\prime}(Y)+\ldots \\
& P=P_{0}+P_{1} x+\frac{1}{2} P_{2} x^{2}+\frac{1}{2} P_{3} z^{2}+\ldots
\end{aligned}
$$

The key assumption here is that at $x-z=0$ the solution is generally non-differentiable about a small elliptic region centred about $x-z=0$. The parameter $\mu$ is essentially a lengthscale parameter, whilst $\lambda$ is a measure of the aspect ratio of the elliptic region (which may also be related to the relative magnitude of the streamwise and crossflow velocities, close to the surface). Notice that (3.7)-(3.10) sat isfy the cont inuity equation (3.3).

In order that the appropriate boundary conditions on $y-0$ are sat isfied, we must have

$$
\begin{aligned}
& v_{0}(0)=v_{0}^{\prime}(0)=0 \\
& v_{1}(0)=v_{1}^{\prime}(0)=0 \\
& \left.w_{1}(0)=w_{1}^{\prime}(0)=0 \quad \text { (and hence } v_{1}^{\prime \prime}(0)=0\right) \\
& w_{2}(0)=v_{2}(0)=0
\end{aligned}
$$




$$
\begin{aligned}
& v_{2}{ }^{\prime}(0)-\mu^{2} \lambda^{2} u_{0}{ }^{n}(0) \\
& u_{23}(0)=-\frac{1}{2} \mu^{2} u_{0}{ }^{n}(0)
\end{aligned}
$$

The key assumptions here, at least from the physical point of view, are equations ( $3.11 a)$ and $(3.11 c)$ (the remainder of these conditions are basically zero velocity restraints). Equation ( 3.11 a) demands that at $x-z=0$ the streamwise component of wall strear vanishes (c.f.I and II), whilst (3.11c) demands that the $z$ derivative of cross flow wall shear vanishes, simultaneously, at this point.

Since we expect the solution to be regular as $Y \longrightarrow 0$, we may expand the solution in the form of a Taylor series in this $11 \mathrm{mit}$, for example

$$
\begin{aligned}
& U_{0}(Y)=\sum_{n=2}^{\infty} \frac{a_{n} Y n}{n !}, \\
& w_{1}(Y)-\sum_{n=2}^{\infty} \frac{c_{n} Y n}{n !} .
\end{aligned}
$$

It is a simple matter to show that

$$
v_{1}(Y)=-U_{0} \int_{0}^{Y} \frac{U_{0} w_{1}-P_{1}+U_{0}}{U_{0}^{2}} d Y .
$$

However we expect $V_{1}$ to be regular as $Y \longrightarrow 0$ also, and this condition then also demands

$$
\begin{aligned}
& a_{2}=U_{0}{ }^{\prime}(0)=P_{1}, a_{3}-U_{0} \cdots(0)-0, \\
& a_{4}=U_{0} \cdots(0)=0, \quad u_{0} \cdots \cdots(0)-a_{3}-0 .
\end{aligned}
$$

A similar, local analysis with the $z$ momentum equation, as $Y \longrightarrow 0$, reveals the, solution to remain regular provided, the following additional conditions are fulfilled

$$
\begin{aligned}
& c_{2}=w_{2} \cdot(0)-P_{3}, c_{3}=0 . \\
& c_{4}=w_{2} \cdots \cdots(0)-c_{2}{ }^{2} .
\end{aligned}
$$

(A similar analysis is also possible vith $i_{2}$ and $v_{2}$. aithough we 
have more than enough details to proceed to the next stage of the anlaysis).

At this stage it is interesting to distinguish between the above, 1 and 11. Setting $z-0, w_{1}-w_{2}-\ldots-0$, togther with $\mu-1$ in the above reduces to a system identical to that described in 1 . The key difference with the work of II is that in the latter, no restriction was placed on $w_{1}^{\prime}(0)$; the primary repercussion of this was that unlike the above (and I), the no-slip condition downstream $(x>0)$ was violated, and so an inner, Goldstein type layer was found necessary. This leads to a situation, in general, where the downstream wall shear is no longer symmetrical about $x=0$ (as in the case in the above and 1 ). A further difference in 11 was that the discontinuity in wall shear was centred along a line $(x-0)$, (rather than at a point, as above), thus the present structure is rather more three-dimensional. A further important distinction is described later.

It is interesting to note that conditions $(3.11 \mathrm{a})-(3.11 \mathrm{c})$ imply that at $x-z-0$, all components of the vorticity vector on the wall are zero, and this is exactly the condition for a point of separation as described by Lighthill (1963). Thus the situation described above is important generically.

Unfortunatly, due to the limited literature available on integrating the three-dimensional boundary layer equations (even of a non-interacting nature), there do not appear to be any calculations illustrating the above characteristics. (Cebeci et al 1980 did present an example of flow past the special case of a paraboloid of zero thickness, with a point where $\mathrm{v}_{0}{ }^{\prime}(0)=0$, but with $w_{1}{ }^{\prime}(0)+0$; this provided the stimulus for the work of 11 .)

The discontinuity in slope of the solution here at the origin 
(and in I and II) is removed by mearis of a triple deck, centred at $x-y=z-0$, and we focus our attention on this aspect next. 


\section{THE INTERACTING REGION}

As in I and II, the appropriate streamise scale is $x-0(\delta)$, where $\delta-R^{-1 / s}$. We also expect the appropriate crossflow lengthscale to be the same, namely $z-0(\delta)$, and so scaled variables $(X, Z)$ are defined by

$$
\begin{aligned}
& x=x / \delta \\
& z=z / \delta
\end{aligned}
$$

Consider first the main deck, comparable in thickness to the classical boundary layer described in the previous section.

The expansion for the solution in this $(Y-O(1)$ ) region (guided partly by $(3.7)-(3.10)$, and partly by 1 and 11$)$ is

$$
\begin{aligned}
& u-U_{0}(Y)+\delta\left\{-X\left[w_{1}(Y)+V_{1}^{\prime}(Y)\right]+A(X, Z) U_{0}^{\prime}(Y)\right\}+\delta^{2}\left\{-\frac{1}{2} X^{2}\left[V_{2}^{\prime}(Y)+w_{2}(Y)\right\}\right. \\
& +\frac{1}{2}\left[A(X, Z)^{2}+\gamma(Z)\right] U_{0}{ }^{n}(Y) \\
& \left.+Z^{2} u_{23}(Y)-X A(X, Z)\left[V_{1}^{n}(Y)+w_{1}^{\prime}(Y)\right]\right\}+\ldots \\
& V=V_{1}(Y)-A_{X} U_{0}(Y) \\
& +\delta\left\{X V_{2}(Y)-A_{X} U_{0}^{\prime}(Y)+(X A)_{X}\left(V_{1}^{\prime}(Y)+w_{1}(Y)\right)-(Z A)_{Z} w_{1}(Y)\right\} \\
& +\ldots \\
& w-\delta\left[Z w_{1}(Y)\right]+\delta^{2}\left\{X Z w_{2}(Y)+Z A(X, Z) w_{1}(Y)\right\}+\ldots \\
& P=P_{0}+\delta P_{1} X+\frac{1}{2} \delta^{2} P_{2} X^{2}+\frac{1}{2} \delta^{2} P_{3} Z^{2}+\delta^{5 / 2} \widetilde{P}(X, Z)+\ldots
\end{aligned}
$$

The function $\gamma(Z)$ here is an arbitary function of $Z$, which as we shall see performs a key role in the investigation, and is analogous to the constant $\Gamma$ in $1 . \gamma(Z)$ is a function we may postulate, although we shall assume $\gamma-o\left(Z^{2}\right)$ as $Z \longrightarrow \propto$ in order that a proper match is made with the results of the previous section. The functions $A(X, Z)$ and $\bar{P}(X, Z)$ are a displacement function and pressure perturbation respectively, and arise from the interaction to be described shorily.

In order that a proper match is made with $(3.7)-(3.10)$ we requite 


$$
A^{2}(X, z)-\mu^{2}\left(\lambda^{2} x^{2}+z^{2}\right) \text { as } x^{2}+z^{2} \rightarrow \infty
$$

The system (4.3)-(4.6) clearly violates the no-slip condition on $Y=0$, and hence the inclusion of a lower deck is necessary. In order that a meaningful balance of terms is affected, this must have thickness given by

$$
\bar{Y}=Y \delta^{-1 / 4}=0(1) \text {, }
$$

(the same thickness as found in I and II). In this region, the solution expansion given in (4.3)-(4.5) remains essentially unchanged, except $Y$ is replaced by $\delta \dot{t} \bar{Y}$, and there is a correction to the $u$ expansion of $\delta^{2} \bar{U}(X, \bar{Y}, Z)$, to the $V$ expansion of $\delta^{5 / 4} \tilde{V}(X, \bar{Y}, Z)$ and to the w expansion of $\delta^{2} \widetilde{W}(X, \bar{Y}, Z)$. The governing equations for these correction terms are

$$
\begin{aligned}
& \frac{\partial \tilde{U}}{\partial X}+\frac{\partial \tilde{V}}{\partial \bar{Y}}+\frac{\partial \bar{w}}{\partial Z}=0, \\
& \frac{1}{2} a_{2} \overline{\mathrm{Y}}^{2} \frac{\partial \overline{\mathrm{U}}}{\partial \mathrm{X}}+\mathrm{a}_{2} \overline{\mathrm{Y}} \overline{\mathrm{V}}=-\frac{\partial \overline{\mathrm{P}}}{\partial \mathrm{X}}+\frac{\partial^{2} \tilde{\mathrm{U}}}{\partial \overline{\mathrm{Y}}^{2}}, \\
& \frac{1}{2} a_{2} \bar{Y}^{2} \frac{\partial \tilde{w}}{\partial x}=\frac{\partial^{2} \tilde{w}}{\partial \tilde{Y}^{2}}-\frac{\partial \tilde{P}}{\partial z}
\end{aligned}
$$

where $a_{2}=U_{0}{ }^{\prime \prime}(0)$.

The boundary conditions to be applied to this system are that

$$
\overline{\mathbf{U}}, \overline{\mathrm{W}} \rightarrow 0 \text { as } \overline{\mathrm{Y}} \rightarrow \infty,
$$

together with

$$
\begin{aligned}
& \bar{W}(\bar{Y}-0)-\bar{V}(\bar{Y}=0)-0 \\
& \bar{U}(\bar{Y}-0)=-\frac{a_{2}}{2}\left\{A^{2}+\gamma(Z)-\mu^{2}\left(\lambda^{2} x^{2}+Z^{2}\right)\right\} .
\end{aligned}
$$

Differentiating (4.10) with respect to $x$, and $(4.11)$ with respect to $Z$, and adding yields

$$
\begin{aligned}
& \frac{1}{2} a_{2} \bar{Y}^{2}\left(\frac{\partial^{2} \bar{U}}{\partial X^{2}}+\frac{\partial^{2} \bar{w}}{\partial X \partial Z}\right)+a_{2} \bar{Y} \frac{\partial \bar{V}}{\partial X} \\
& =-\frac{\partial^{2} \bar{P}}{\partial X^{2}}-\frac{\partial^{2} \bar{P}}{\partial Z^{2}}+\frac{\partial^{3} \bar{U}}{\partial Y^{2} \partial X}+\frac{\partial^{3} \bar{W}}{\partial \bar{Y}^{2} \partial Z}
\end{aligned}
$$


Invoking cont inulty (4.9), and then differentlating with respect to $\bar{Y}$ yields

$$
\frac{\partial^{4} \bar{V}}{\partial \bar{Y}^{4}}-\frac{1}{2} \quad a_{2} \bar{Y}^{2} \quad \frac{\partial^{3} \bar{v}}{\partial \bar{Y}^{2} \partial x}+a_{2} \frac{\partial \tilde{v}}{\partial x}=0
$$

Here the problem has been reduced to a (quasi-) two-dimensional problem, which greatly simplifies obtaining a solution. The boundary conditions to be imposed are: on $\bar{Y}-0$

$$
\begin{aligned}
& \tilde{\mathrm{v}}=0, \\
& \frac{\partial \overline{\mathrm{V}}}{\partial \bar{Y}}=\frac{1}{2} \mathrm{a}_{2}\left[2 \mathrm{AA} X-2 \mu^{2} \lambda^{2} \mathrm{X}\right], \\
& \frac{\partial^{3} \overline{\mathrm{v}}}{\partial \overline{\mathrm{Y}}^{3}}=-\frac{\partial^{2} \overline{\mathrm{p}}}{\partial \mathrm{X}^{2}}-\frac{\partial^{2} \tilde{\mathrm{P}}}{\partial \mathrm{Z}^{2}}
\end{aligned}
$$

whilst $\quad \tilde{\mathbf{V}} \rightarrow 0$ as $\overline{\mathbf{Y}} \rightarrow \infty$.

A very similar system was considered by Stewartson (1970), the key result of which shows that a solution subject to $(4.16)$ is only possible if

$$
\begin{aligned}
& \frac{1}{2} \quad a_{2}\left[A^{2}+\gamma(Z)-\mu^{2}\left(\lambda^{2} x^{2}+z^{2}\right)\right] \\
& =-\frac{\left(\frac{1}{4} !\right)}{2^{3 / 2}\left(\frac{1}{4} !\right)} \int_{-\infty}^{x} \frac{\partial \overline{\mathrm{p}} / \partial \xi+\int_{-\infty} \frac{\partial^{2} \overline{\mathrm{p}}}{\partial \mathrm{Z}^{2}} \mathrm{~d},}{(X-\xi)^{\frac{1}{2}}} \mathrm{~d},
\end{aligned}
$$

(subject to $(4.7))$.

The system is now closed by consideration of the upper deck, wherein $Y=O\left(\delta^{-3 / 2}\right)$. The solution is standard, with the pressure being determined by Laplaces equation, and so following Smith et al (1977) and Duck and Burggraf (1986) we may write

$\bar{p}(x, z)=-\frac{1}{2 \pi} \quad U_{0}(\infty)^{2} \int_{-\infty}^{\infty} \int_{-\infty}^{\infty} \frac{\partial^{2} A / \partial \xi^{2} d \xi d \zeta}{\left[\left(x^{\prime}-\xi\right)^{2}+(z-\zeta)^{2}\right]^{\frac{1}{2}}}$.

The combination of (4.17) and (4.18) represents a closed problem, the 
solution of which will be discussed in the following section.

We note that the condition (4.7), as $x^{2}+z^{2} \longrightarrow \propto$ can be made stronger, namely

$$
A^{2}(X, z)-\mu^{2}\left(\lambda^{2} X^{2}+z^{2}\right)-\gamma(z)
$$

This is analogous to the farfield condition found in $I$, namely

$$
A^{2}(x)-x^{2}-\Gamma
$$

(where $\Gamma$ is a constant). We note that if $\gamma(z)$ is entirely negative, then we might expect there to be no difficulty in obtaining a solution to the system described above, with the flow remaining ent irely at tached. However as $\gamma(Z)$ increases, and becomes progressively more positive, (4.19) suggests a three-dimensional analogue to the classical Coldstein square-root singularity is possible, leading to a catastrophic breakdown. (Indeed, similar observations are noted in the work of I and II.) One of the primary aims of the following sections will be to determine the maximum value of $\gamma(Z)$ for which solutions exist. Notice, also, that as $\gamma(Z)$ becomes progressively more negative the effect of the interaction is reduced, and (4.19) becomes an asymptotic solution to the above system.

In II it was assumed that the cross-stream pressure gradient was known a priori, (not a completly rational assumption) leading to a one-dimensional mathematical problem, similar to that found in 1 ; here, the mathematical problem $(4.17)-(4.18)$ is fully two-dimensional. 


\section{NUMERICAL TECHNIOUE}

At this stage it is convenient to take the double Fourier Transform of (4.17) and (4.18) to eliminate the pressure perturbation $\bar{P}(X, Z)$.

Defining

$$
A^{* *}-\int_{-\infty}^{\infty} \int_{-\infty}^{\infty} A(x, z) e^{-i k x-i \ell z} d x d z
$$

(with other transform quant it les defined similarly), then (4.17) and (4.18) give

$$
\begin{aligned}
\frac{1}{2} \text { a }_{2} & {\left[A^{2}+\gamma(Z)-\mu\left(\lambda^{2} X^{2}+Z^{2}\right)\right]^{\star \star} } \\
= & -\frac{\left(-\frac{1}{4} !\right)}{2\left(\frac{1}{4} !\right)}\left(\frac{\pi}{2}\right)^{\frac{1}{2}} \quad(i k)^{\frac{1}{2}}\left(k^{2}+\ell^{2}\right)^{\frac{1}{2}} U_{0}(\infty)^{2} A^{\star \star} .
\end{aligned}
$$

This equation can be transformed back into $(X, Z)$ space, namely

$$
A(x, z)=-\Lambda \int_{-\infty}^{\infty} \int_{-\infty}^{\infty} \frac{L(\xi, \zeta) \mathbf{K}(x)}{\left[(x-\xi)^{2}+(z-\zeta)^{2}\right]^{2}} d \xi d \zeta
$$

where $K(X)$ denotes the complete elliptic integral of the first $k$ ind, argument $x$, where

$$
x=\left[1+\frac{x-\xi}{\left[(x-\xi)^{2}+(2-5)^{2}\right]^{\frac{1}{2}}}\right]^{\frac{1}{2}}
$$

and

$$
L(X, Z)=\frac{1}{2} \text { a } 2\left[A^{2}+\gamma(Z)-\mu^{2}\left(\lambda^{2} X^{2}+Z^{2}\right)\right]
$$

and

$$
\Lambda=\frac{8\left(\frac{1}{4} !\right)}{\pi^{3 / 2}\left(-\frac{1}{4} !\right) U_{0}(\infty)^{2}}
$$

However from a numerical point of view (5.3) would present a number of difficulties, primarily associated with singularities in the kernel term. Instead we prefer to adopt an alternative scheme based on the transform equation (5.2). If we witite 
$(A, \gamma, X, Z, k, \ell)-\left(\beta \mu^{3 / 5} \hat{A}, \beta^{2} \mu^{6 / 5} \gamma, \beta \mu^{-2 / 5 x}, \beta \mu^{-2 / 5} Z, \beta^{-1} \mu^{2 / 5} \hat{k}, \beta^{-1} \mu^{2 / 5} \mathcal{C}\right)(5,7)$

where

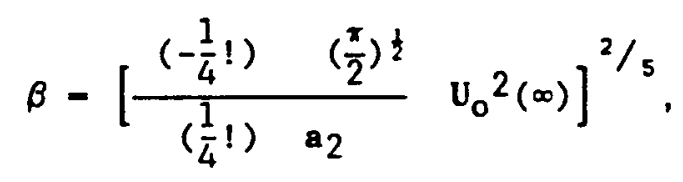

then (5.2) simplifies to

$$
\begin{aligned}
{\left[\hat{A}^{2}\right.} & \left.+\hat{\gamma(z)}-\left(\hat{\lambda}^{2} \hat{x}^{2}+\bar{z}^{2}\right)\right]^{\star *} \\
& =(\hat{i k})^{\frac{1}{2}}\left(\hat{\mathrm{k}}^{2}+\hat{\mathrm{Q}}^{2}\right)^{\frac{1}{2}} \hat{A}^{2 k *} .
\end{aligned}
$$

In its present form (5.9) is unsultable for numerical manipulations, because of unboundedness as $\hat{x}^{2}+\hat{z}^{2} \longrightarrow \infty$ (this is mirrored in $\hat{k}, \hat{l}$ space by a singularity as $\hat{k}$ and/or $\hat{l} \longrightarrow 0$ ). We do, however have the condition

$$
\hat{A}^{\hat{2}}-\lambda^{2} \hat{x^{2}}+\hat{z^{2}}-\hat{\gamma}(\hat{z}) \text { as } \hat{x^{2}}+\hat{z}^{2} \longrightarrow \infty \text {. }
$$

Cuided by this we write

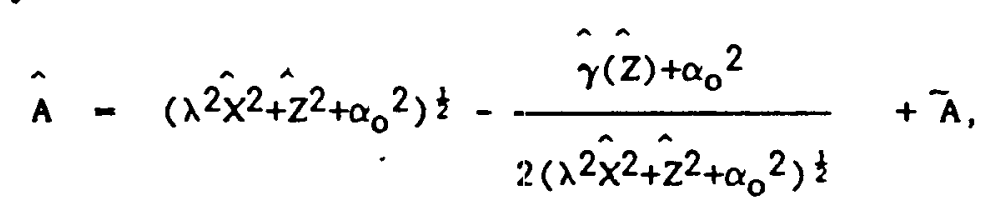

where now we have $\bar{A} \longrightarrow 0$ as $\hat{x}^{2}+\hat{Z}^{2} \longrightarrow \infty$. We have introduced the (artificial) constant $\alpha_{0}$ into the problem in order to ensure boundedness as $\hat{x}^{2}+\hat{z}^{2} \longrightarrow 0$. (This is mirrored in $\hat{k}, \hat{l}$ space by appropriate decay as $\hat{\mathrm{k}}^{2}+\hat{\ell}^{2} \longrightarrow \infty$; setting $\alpha_{0}=0$ would cause difficulties with the transform in this limit.) $\alpha_{0}$ may take on any real value, and is thus a parameter entirely of our choice, the exact solution for $\hat{A}$ being completely independent of $a_{0}$.

Substituting (5.11) into (5.9) yields

$$
\begin{aligned}
& \left\{\frac{\gamma(Z)+\alpha_{0}^{2}}{4 r^{2}}+2 \bar{A} r+\bar{A}^{2}-\frac{\bar{A}\left[\gamma(Z)+\alpha_{0}^{2}\right]}{r}\right\}^{\star *} \\
& =(i k)^{\frac{1}{2}\left(k^{2}+C^{2}\right) \frac{1}{2}}\left\{r-\frac{\gamma(Z)+\alpha_{0}^{2}}{2 r}+\bar{A}\right\}^{* *}
\end{aligned}
$$


Here, for convenience we have written

$$
r-\left(\lambda^{2} \hat{x}^{2}+\hat{z}^{2}+\alpha_{0}^{2}\right) \frac{1}{2} \text {. }
$$

Unfortunately (5.13) is still not quite suitable for numerical

treatment (because of difficulties with the second term on the left-handside of $(5.13)$ as $\left.x^{\hat{2}}+\hat{z}^{\hat{2}} \longrightarrow \infty\right)$. This may be rectified however by writing

$$
\tilde{A}-A_{0}+B,
$$

where

$$
\left[2 A_{o} r\right]^{\star *}-(1 k)^{\frac{1}{2}}\left(k^{2}+l^{2}\right)^{\frac{1}{2}}\left\{r-\frac{\left[\gamma(z)+\alpha_{0}^{2}\right]}{2 r}\right\}^{\star \star} .
$$

Details of the evaluation of $A_{0}$ are given in the Appendix, but now we may regard $A_{0}$ as a known function of $\hat{x}$ and $\hat{z}$ (or $A_{0}{ }^{*}$ as a known function of $\hat{k}$ and $\hat{\ell}$ ), and $B$ the function to be determined.

Equation (5.12) may now instead be written

$$
\begin{aligned}
& \left\{\frac{\left[\gamma(Z)+\alpha_{0}^{2}\right]^{2}}{4 r^{2}}+2 B_{2} r-\left[\gamma(Z)+\alpha_{o}^{2}\right]\left[\frac{A_{0}}{r}+\frac{B}{r}\right]+A_{0}^{2}+2 A_{o} B+B^{2}\right\}^{* \star} \\
& -(\hat{i k})^{\frac{1}{2}}\left(\hat{k}^{2}+\hat{C}^{2}\right)^{\frac{1}{2}}\left(A_{O}+B\right) * * .
\end{aligned}
$$

Equation (5.16) is cast in $\hat{k}, \hat{l}$ space; however although the righthand-side of this equation is most simply evaluated in its present form, in Fourier space, the left-hand-side is best evaluated in physical space. Consequent ly we choose to adopt a solution procedure which exploits the favourable aspects of both spaces. More specifically, the technique is as follows.

For a given $\hat{\gamma}(\hat{Z}), A_{0}$, then $A_{0}{ }^{\star *}$ and the inverse of (ii) $\left(\hat{k}^{2}+\hat{l}^{2}\right)^{\frac{1}{2}} A_{0}{ }^{\star \star}$ are all evaluated (see Appendix for details). Then

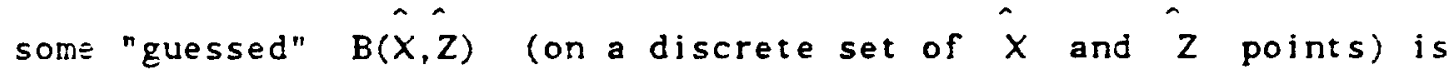
iransformed into $\hat{k}, \hat{l}$ space to yield $B^{\star \star}(\hat{k}, \hat{l})$. This is achieved using the Fast Fourier Transform (F.F.T.) rechnique of Cooley and Tukey (1965). The 
term $(\hat{i k})^{\frac{1}{2}}\left(\hat{k}^{2}+\hat{l}^{2}\right)^{\frac{1}{2}} B^{* *}$ is then constructed, and transformed back to physical space (again by means of the F.F.T. technique). This yields an est imate for the right-hand-side of (5.16) on the $\hat{x}, \hat{z}$ grid, and provides a means of obtaining a new estimate for $B(\hat{X}, \hat{Z})$. A number of different schemes were ultilised for this, but the most reliable, generally, (although not always the scheme that gave the most rapid convergence) was to write (5.16) at each $\hat{x}, \hat{z}$ location as

$$
\mathbf{B}-\mathbf{R}-\alpha_{1} \mathbf{B}^{2}
$$

and to use this to provide a new estimate for $B$, treating the right-handside of this equation as known (some under-relaxation was applied if necessary). In certain regions of parameter space, this iteration procedure was modified slightly by using (5.17) at all $\hat{x}, \hat{z}$ stations, except at one (prescribed) station, $\left(\hat{x}_{0}, \hat{z}_{0}\right)$ say, $\hat{B}\left(\hat{x}_{0}, \hat{z}_{0}\right)$ was prescribed and $\hat{\gamma}\left(\hat{Z}_{0}\right)$ was treated as the unknown (a similar tactic was followed in 1). The process was repeated unt 11 the maximum change to the solution at any of the $X, Z$ points fell below some predetermined tolerance $\left(10^{-5}\right)$. Since we are concerned with flows which are symmetric with respect to $Z=0$, we need only consider $Z<0$. Further, notice that if we use a gridsize in $\hat{x}$ of $\hat{x}$, with $N_{x}$ points, the grid in $\hat{k}$ space is given by

$$
\hat{\Delta \mathrm{k}}=2 \pi / \mathrm{N}_{x} \hat{\Delta \mathrm{X}} \text {. }
$$

This relationship arises through the use of the F.F.T. A similar relationship applies in the crossflow direction,

$$
\Delta \hat{Q}=2 \pi / \mathrm{N}_{\mathrm{Z}} \hat{\Delta Z}
$$

where $\Delta \hat{Z}$ is the gridsize in the $\hat{z}$ direction, $N_{z}$ the number of grid points, and $\hat{\Delta}$ the gridsize in $\hat{\mathscr{C}}$ space.

In the following section we go on to describe a number of results. 


\section{RESULTS AND DISCUSSION}

As a check of the general numerical technique, the two-dimensional case as considered in $J$ was recomputed, using basically the same technique as described in the previous section. This involved solving (5.9) with $\hat{Z}=0$ $\hat{\ell}=0, \lambda-1, \hat{\gamma(z)}=\Gamma$ (a constant), with just a single Fourier Transform (in the $\hat{x}$ direction). Additionally, the particular details, given in the Appendix are rather different, although the overall scheme was similar.

The two-dimensional case was computed with $N_{x}=64, \hat{\Delta x}=0.317$, and $\alpha_{0}=3$; the variation of $\hat{A}(\hat{X}-0)$ with $\Gamma$ is shown in Fig.1. The value of $\hat{A}$ here differs by a multiplicative factor $x^{1 / 2}$ from that of 1 , and the value of $\Gamma$ here by a multiplicative factor $\pi^{2 / 5}$ from that of I (on account of the slightly different normalisation used); to facilitate comparison, Fig.1 also indicates the values corresponding to the normalisation of 1 (these are denoted by a subscript 1 ). The present results reproduce those of $I$, to within graphical accuracy. The non-uniqueness of the solution over a range of $\Gamma$, together with the non-existance of solution for $\Gamma \geq 1.74$ is immediately apparent. On the "lower branch" of the curve, computations became progressively more awkward as $\Gamma$ was reduced, even using the alternative procedure where $\Gamma$ itself is determined during the course of the iteration (although no really exhaustive at tempt was made to extend these results further, the accuracy of the scheme having been confirmed).

We now turn to consider a number of results for the three-dimensional case. To be specific, we shall focus attention on functions $\gamma(Z)$ of the class

$$
\hat{\gamma}(\hat{z})=\Gamma \epsilon^{-\hat{z}^{2}}
$$

and seel. to determine the variation of solutions with $\Gamma$. (Note tha: 
even though this choice of $\hat{\gamma}(\hat{z}) \longrightarrow 0$ as $\hat{z} \longrightarrow \infty$, the results will nonetheless be fully three-dimensional as seen by the form of the far field conditions (4.19)).

Variations of $\hat{A}(\hat{X}, \hat{Z})$ (at selected $\hat{X}, \hat{Z}$ stations) are shown in Fig. 2 . These results were determined with the grid $N_{z}=N_{x}-64, \hat{\Delta x}-\hat{\Delta z}-0.317$, and $\alpha_{0}-3$, for the particular case $\lambda-1$. These distributions are qualitatirely similar to those obtained for the two-dimensional case, although the range of $\Gamma$ for which solutions exist is much increased. To assess the effect of numerical grid changes, Fig.3. shows results for $\hat{A}(\hat{X}-0, \hat{Z}-0)$, obtained using: $\alpha_{0}-3, N_{x}-N_{z}-32, \hat{\Delta x}=\hat{\Delta Z}=0.645$ (broken line), $\alpha_{0}=2, N_{x}-N_{z}=64, \Delta \hat{x}-\hat{\Delta Z}-0.317$ (solidus), and $\alpha_{0}=3$, $\mathrm{N}_{\mathrm{X}}=\mathrm{N}_{\mathrm{Z}}=16, \hat{\Delta x}=\hat{\Delta Z}-0.667$ (dot-dashed line).

It is heartening that there appears to be no difference (to within graphical accuracy) between the $\alpha_{0}-3$ and corresponding $\alpha_{0}=2$ results (indeed the computation was also carried out with $\alpha_{0}-2$, $N_{x}=N_{z}=32, \hat{\Delta x}-\hat{\Delta Z}=0.645$, and this too gave results indistinguishable on the graphical scale used with the corresponding $\alpha_{0}=3$ calculation). On the whole the computations agree very well, although the most prominant discrepancy occurs in the "nose" region of the curve, as may be anticipated, although elsewhere the agreement is to within the accuracy of the figure. The computations on the "lower branch" became progressively more difficult as $\Gamma$ was reduced (a typical computation on the finest, most extensive grid taking of the order of ten minutes on a Cray $X M P)$, and so the curves are not extended as far as the corresponding two-dimensional case (Fig.1).

Figure 4 shows the contours of constant $\hat{A}(\hat{X}, \hat{Z})$ for $\Gamma=2.803103$ (upper branrh) and for comparison Fig. 5 shows the contours for the same value of $\Gamma$, but corresponding to the lower branch (both sets of resulis were obtained using the same grid as used for $F i g, 2)$. Since $\hat{A}(\hat{X}, \hat{Z})$ 
is proportional to the streamwise wall shear, negative $\hat{A}(\hat{X}, \hat{Z})$ implies reversed flow. Comparing these two figures reveals the lower branch to involve a much larger region of reversed flow. Indeed, as the lower branch is followed, with $\Gamma$ reducing, the region of reversed flow is expected to increase without bound (a feature observed in previous two-dimensional studies). As $\hat{x}^{2}+\hat{z}^{2}$ increases the contours became progressively more circular in both cases.

Figure 6 shows the variation of $\hat{A} \hat{(X}-0, \hat{Z}=0)$ with $r$ for the case $\lambda=2$ (obtained using the grid $N_{X}=N_{Z}-64, \hat{\Delta x}-\hat{\Delta Z}-0.317$, and $\left.\alpha_{0}-3\right)$. This case corresponds to a basic flow with a rather stronger streamwise flow near the wall compared with the crossflow. In this case the range of $\Gamma$ over which the solution exists is increased over the $\lambda=1$ results, as is the range of $\hat{A}(0,0)$. Figure 7 . shows contours of constant $\hat{A}(\hat{X}, \hat{z})$ for $\Gamma=5.342597$ (lower branch). The contours as $\hat{X^{2}} \hat{+} z^{2} \longrightarrow \propto$ in this case will no longer approach the circular form of $\lambda=1$, but rather will tend to ellipses.

These results presented here clearly 11 lustrate the occurrence of fully three-dimensional solutions involving below - marginal skin friction. The basic solution technique introduced here, involving the usage of a pseudo-spectral method could fairly easily be extended to other classes of flow, for example flows away from lines of symmetry, supersonic threedimensional flows or flows where $U_{0}{ }^{\prime}(0)$ and $W_{1}{ }^{\prime}(0)$ do not vanish simultaneously (such as that studied in II). However the particular details required in obtaining a well behaved "perturbation" solution to compute (c.f. the Appendix) would necessarily have to be modified. Further, and important ly, the theory presented here is not subject to the limitations imposed as a result of the essentially two-dimensional interaction condition employed in 11 . 
lt is worth making a few final comments regarding the choice and a

determination of the function $\gamma(Z)$. In this paper $\gamma(Z)$ represents

a quite natural extension of the constant $\Gamma$ found in the

two-dimensional work of $I$ (and $\Gamma_{ \pm}$in II). In $1, \Gamma$ was interpreted

as being proportional to the excess of the angle of incidence

(or camber) over the critical value. As described in 11 , the

interpretation of the two related constants $\Gamma_{ \pm}$occuring in that study

is less obvious. In the present work, we may interpret $\hat{\gamma(Z)}$ as being

a measure of the excess angle over the critical centreline angle; in the

present context the local angle can depend on spanwise location, and so

this excess will in general be a function of lateral position, which from

a physical point of view can be regarded as a spanwise (i.e. $\hat{Z}$ )

variation of body surface curvature and/or of streamwise conditions (we

must however restrict spanwise variations to be on a scale $z=O(\delta)$

or longer).

Our particular choice of $\hat{\gamma(z)}$, namely (6.1) leads to necessarily

a model problem; however because of the qualitative similarity between the

results for this particular cholce of $\hat{\gamma(Z)}$ and those found in 1 and

II, we fully expect the present three-dimensional results to give a good

indication of the qualitative feature of general flows of this class.

Since $\hat{\gamma(z)}$ represents an "excess" over the critical state,

by its nature it controls the amount of interaction in the problem (see

also the remarks, towards the end of Section 4 regarding this point).

Hence it is not directly deducible from "non-interacting" calculations such

as those of Cebeci at al (1988). (The constant $\Gamma$ found in 1 is also

not directly obtainable from "non-interacting" calculations.) 


\section{Acknowledrements}

The author wishes to thank Professor O.R. Burggraf for encouragement and many useful discussions relating to this work. Computations were carried out at the Ohio Supercomputer Center, the University of Manchester Regional Computer Cent re (with computer time provided under S.E.R.C Grant No. GR/E/25702) and at NASA Lewis Research Center (whilst the author was visiting under the ICOMP program). 


\section{Appendix}

Here we consider the evaluation of $\hat{A}_{0}$ and $\hat{A}_{0}{ }^{\star *}$. Since certain of the components in the evaluation of $\hat{A}_{0}$ are unbounded as $\hat{x}^{2}+\hat{z}^{2} \longrightarrow \infty$ (and this is reflected by singularities as $\hat{k}^{2}+\hat{l}^{2} \longrightarrow 0$ in Fourier space), a certain amount of analytic work is necessary, prior to any numerics. Fortunately, the inclusion of the $\alpha_{0}$ term (see (5.11)) ensures decay in $\hat{k}, \hat{l}$ space as $\hat{k}^{2}+\hat{l}^{2} \longrightarrow \infty$, and so this limit requires no special attention.

By (5.15) we have

$$
\left(r A_{0}\right)^{\star *}-\frac{1}{2}(i k)^{\frac{1}{2}}\left(k^{2}+l^{2}\right)^{\frac{1}{2}}\left\{r-\frac{\left[\gamma(z)+\alpha^{2} 0\right]}{2 r}\right\}^{\star *}
$$

It is straight forward to show

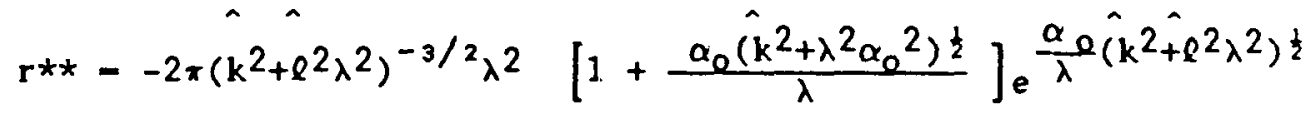

(assuming $\alpha_{0}>0$ ).

The quantity $\left.\mathrm{r}^{* *}(\hat{\mathrm{ik}})^{\frac{1}{2}}{\hat{\left(\mathrm{k}^{2}\right.}}^{2} \hat{l}^{2}\right)^{\frac{1}{2}}$ is transformed back to $\hat{x, z}$ space by the integral

$-\frac{4 \lambda^{2}}{\pi} \int_{0}^{\infty} \int_{0}^{\pi / 2} \frac{(\cos \varphi)^{\frac{1}{2}}}{\Phi^{3}} e^{-\frac{\alpha_{0}}{\lambda} \hat{\rho}^{2} \Phi}\left(1+\alpha_{0} \frac{\rho^{2}}{\lambda} \phi\right) \cos \left(\rho^{2} \cos \varphi X+\frac{\pi}{4}\right) \cos \left(\rho^{2} \sin \varphi \mathrm{Z}\right) \mathrm{d} \rho \mathrm{d} \varphi$

where $\Phi=\left[\cos ^{2} \varphi+\lambda^{2} \sin ^{2} \varphi\right]^{\frac{1}{2}}$.

Here the Fourier variables $\hat{k}, \hat{l}$ have been transformed to Fourier polar variables $\bar{\rho}, \varphi$, and then the radial variable $\hat{\rho}$ is introduced by $\bar{\rho}=\hat{\rho} \bar{t}$. The result, (A.3) is a fairly well behaved integral, which is readily amenable to numerical quadrature (the weak square root singularily at $f=\pi / 2$ was not found to cause any difficulties).

The " $\alpha_{0} 2 / 2 r$ " term in (A.1) was treated in much the same way as above, using the result

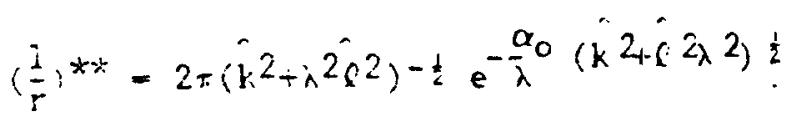


The remaining term was evaluated rather more numerically. We have

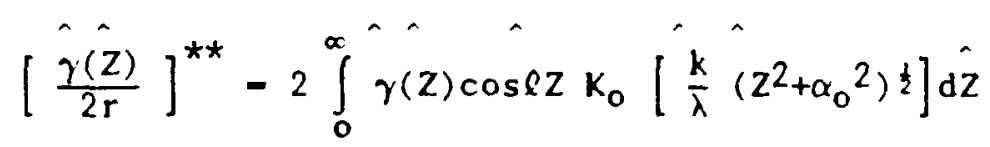

(having carried out the $\hat{x}$ transform. Here $K_{0}(x)$ denotes the modified Bessel function of order zero, argument $\lambda$. The $z$ integration generally requires a numerical treatment (depending on the particular form for $\gamma(z))$. Once evaluated, the quantity (ik) $\frac{1}{2}\left(k^{2}+c^{2}\right) \frac{1}{2}\left\{\frac{\gamma(z)}{2 r}\right\}^{\star \star}$ was then transformed to $(\hat{X}, \hat{z})$ numerically, completing the determination of $A_{0}$. $A_{0}{ }^{*}$ was then evaluated without any special numerical treatment, $(\hat{i k})^{\frac{1}{2}}\left(\hat{k}^{2}+\hat{l}^{2}\right) \frac{1}{2} A_{0} * *$ was constructed, and then $t$ ransformed back to $x, z$ space (again, with no special treatment), thus completing the preliminary computation, prior to the iteration commencing. 


\section{References}

BODONYI, R.J. and DUCK, P.W. 1988 A numerical method for treating strongly interactive three-dimensional vilscous-inviscid flows. Computers and Fluids $16,279$.

BROWN, S.N. 1985 Marginal separation of a three-dimensional boundary layer on a line of symmetry, J Fluid Mech. 158, 95.

BROWN, S.N. and STEWARTSON, K. 1983, On an integral equation of marginal separation SIAM J. Appl. Maths. 43, 1119.

CEBECl, T. and SU, W. 1988. Separation of three-dimensional laminar boundary layers on a prolate spheroid, J. Fluid. Mech. 191, 47. CEBECI, T. KHATTAB, A.K. and STEWARTSON, K. 1980 On nose separation J.Fluid Mech. 97, 435 .

COOLEY, J.W. and TUKEY, J.W. 1965 An algorithm for the machine computation of complex Fourier seiries. Math. Comp. 19, 297.

DUCK, P.W. and BURGGRAF, O.R. 1986 Spectral solutions for three-dimensional triple-deck flow over surface topography. J. Fluid Mech. 162, 1.

FORNBERG, B. 1980 A numerical study of steady viscous flow past a circular cylinder. J.Fluid Mech. $98,819$.

FORNBERG, B. 1985 Steady viscous flow past a circular cylinder up to a Reynolds number 600. J. Comp. Phys. 61, 297.

GOLDSTEIN, S. 1948 On laminar boundary layer flow near a point of separation. Q.J. Mech. Appl. Maths. 1, 43 .

LIGHTHILL, M.J. 1963 'In Laminar Boundary Layers' (ed. L. Rosenhead). Cha.2. Oxford University Press.

PEREGRINE, D.H. 1985 A note on the steady high-keynolds number flow about a circular cylinder. J.Fluid Mech. 157.439. 
SMITH, F.T. 1979 Laminar flow of a incompressible fluid past a bluff body;

the separation, reat tachment, eddy properties and drag. J.Fluid Mech. 92, 171.

SMITH, F.T. 1982 Concerning dynamic stall. Aero Q. 33, 331.

SMITH, F.T. 1983 Properties, and a finite-difference approach, for three-dimensional interactive boundary layers. UTRC Rep. 83-46, United Technical. Res.Cent. East Hertford, Conn.

SMITH, F.T. 1985 A structure for laminar flow past a bluff body at high Reynolds number. J. Fluid Mech. 155, 175.

SMITH, F.T., SYKES, R.I. and BRIGHTON, P.W.M. 1977 A two-dimensional boundary layer encountering a three-dimensional obstacle. J. Fluid Mech $\underline{83}, 163$.

STEWARTSON, K. 1970 Is the singularity at separation removable?

J. Fluid Mech. 44,347

STEWARTSON, K., SMITH, F.T., and KAUPS, K. 1982 Marginal separation.

Stud. Appl. Maths. 67, 45 .

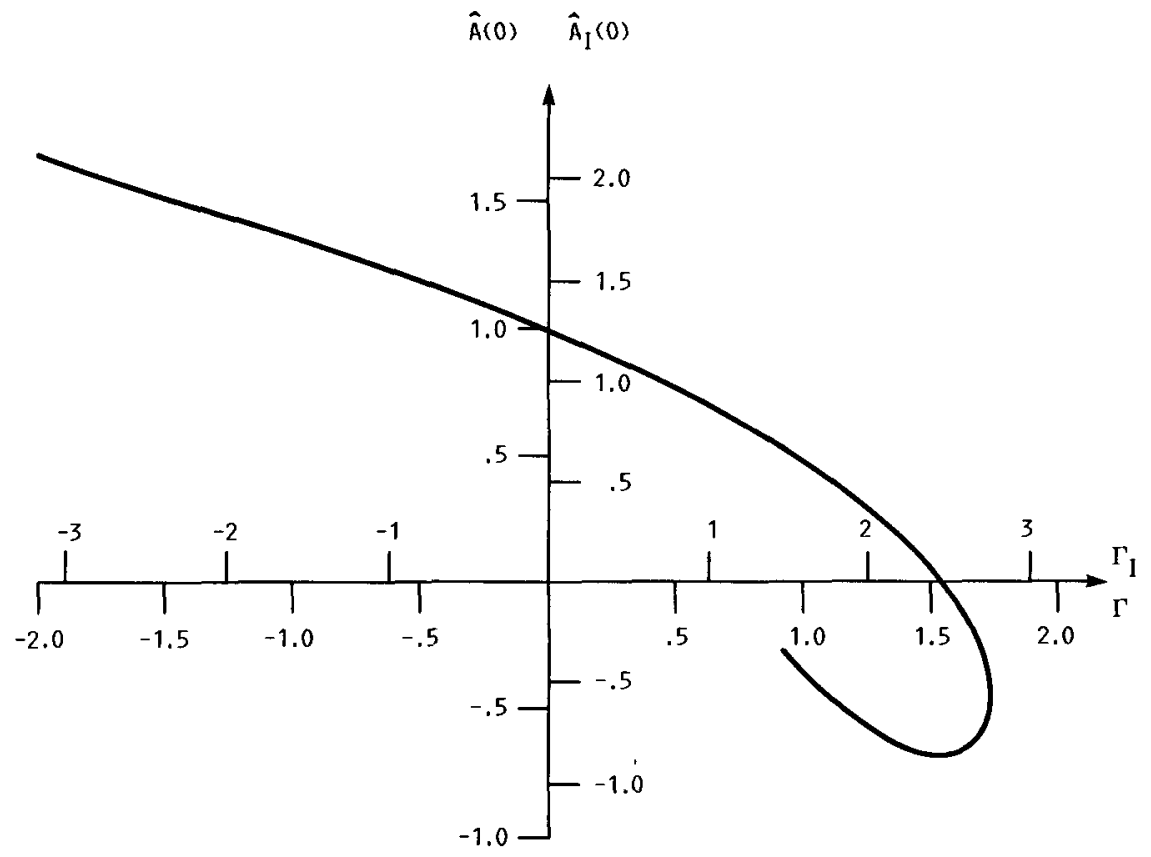

Figure 1. - VARIATION OF $\hat{A}(0)$ WITH $\Gamma$ (TWO-DIMENSIONAL CASE), $\hat{A}_{I}, \hat{\Gamma}_{I}$ DENOTE VALUES USING NORMALIZATION OF I. 


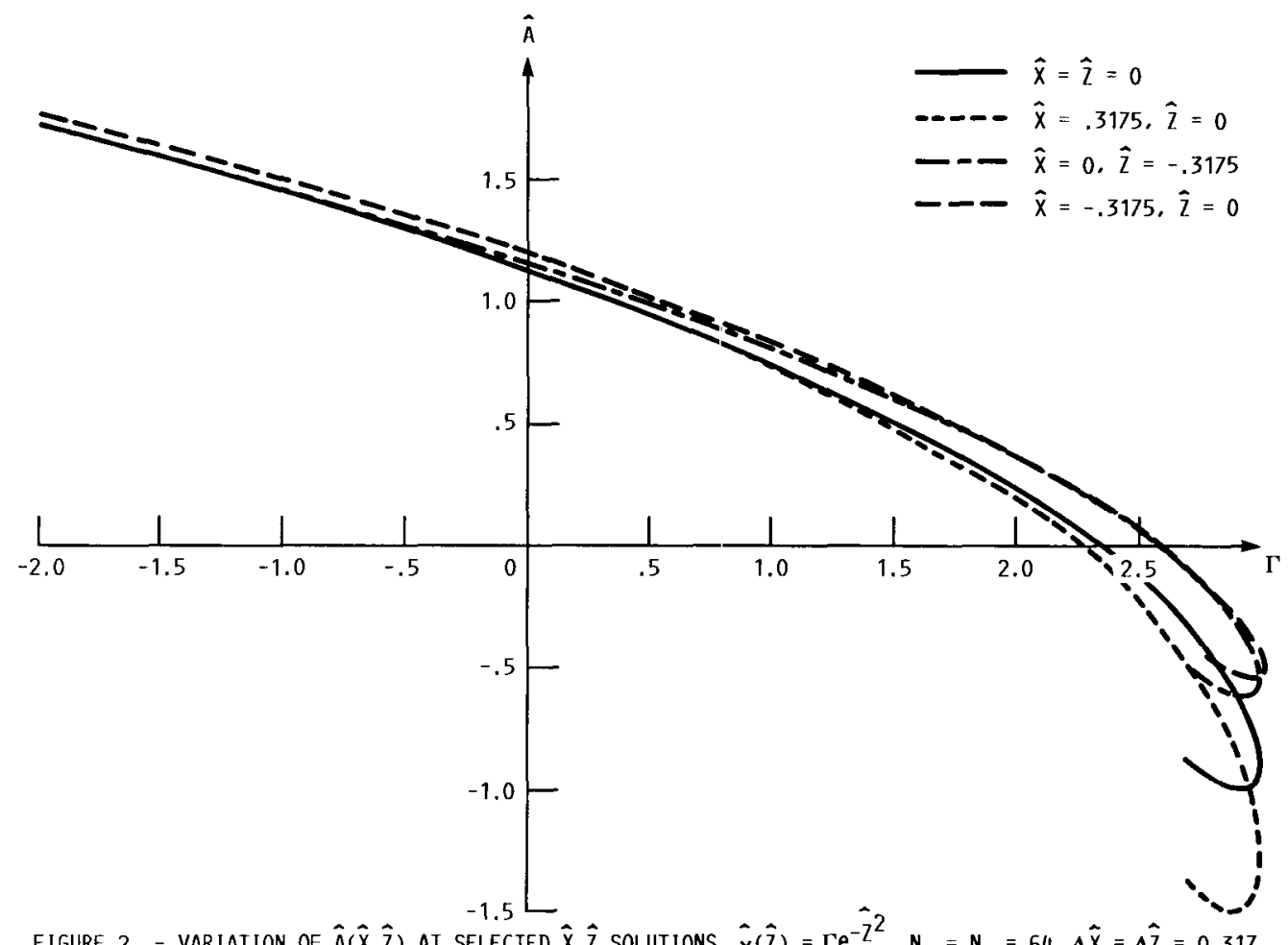

Figure 2. - variation of $\hat{A}(\bar{x}, \hat{z})$ at Selected $\hat{x}, \hat{z}$ solutions, $\hat{\gamma}(\hat{z})=\Gamma e^{-\hat{z}^{2}}, N_{x}=N_{z}=64, \Delta \hat{x}=\Delta \hat{z}=0.317$, $a_{0}=3, \lambda=1$.

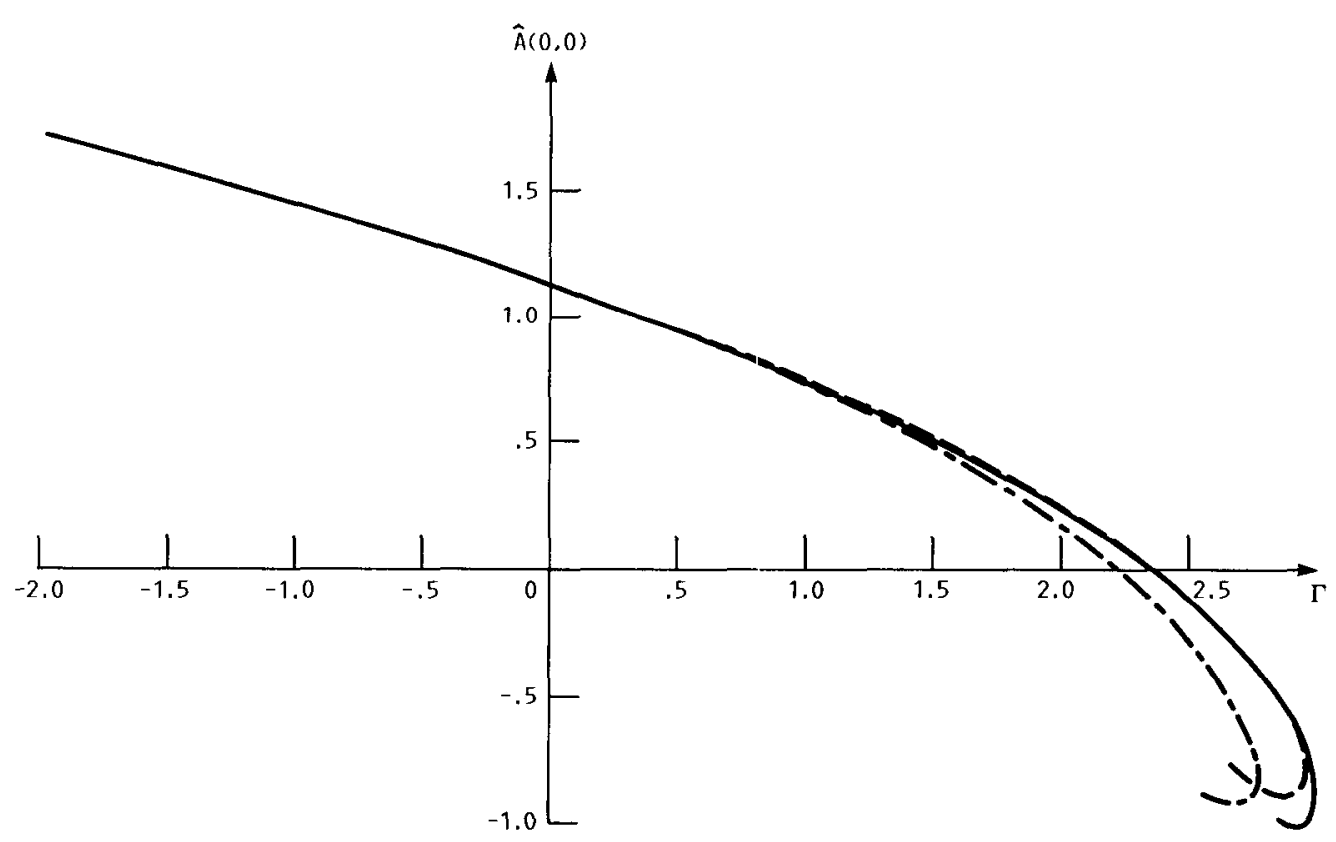

Figure 3. - DETAILS AS ABOVE, BUT $\hat{x}=\hat{z}=0$, AND $N_{x}=N_{x}=64, \Delta \hat{x}=\Delta \hat{z}=0.317, a_{0}=2$ (SOLIDUS), $N_{x}=N_{z}=32, \Delta \hat{x}=\Delta \hat{z}=0.645$ (BROKEN LINE) $N_{x}=N_{z}=16, \Delta \hat{x}=\Delta \hat{z}=0.667, a_{0}=3$ (DOT-DASHED 


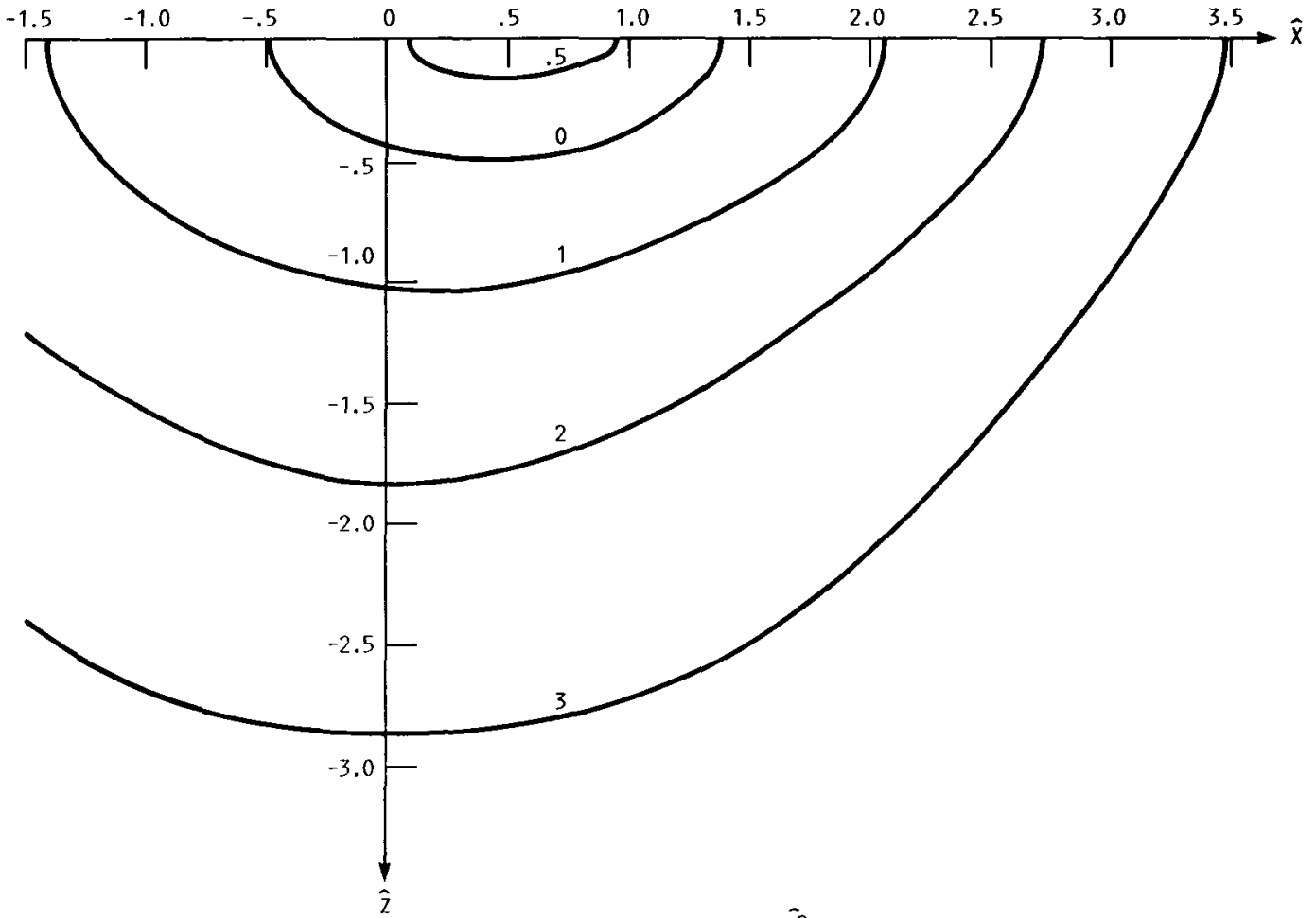

FIGURE 4. - CONTOURS FOR CONSTANT $\hat{A}(\hat{x}, \hat{z}) \hat{y}(\hat{z})=2.803103 \mathrm{e}^{-z^{2}}, \lambda=1$. UPPER BRANCH SOLUTION.

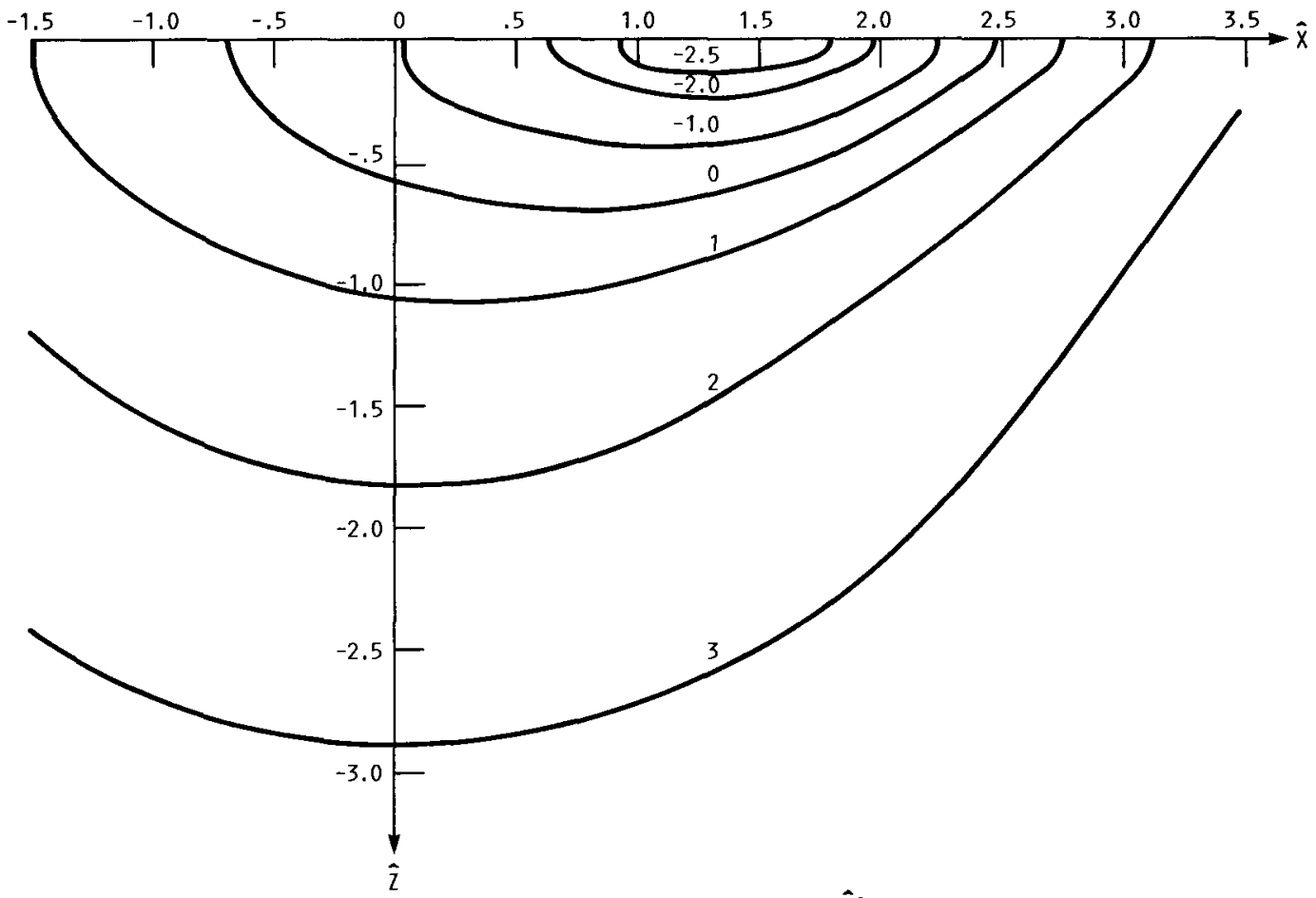

FIGURE 5. - CONTOURS FOR CONSTANT $\hat{A}(\hat{x}, \hat{z}) \cdot \hat{\gamma}(\hat{z})=2.803103 \mathrm{e}^{-\hat{z}^{2}}$, LOWER BRANCH SOLUTION. 


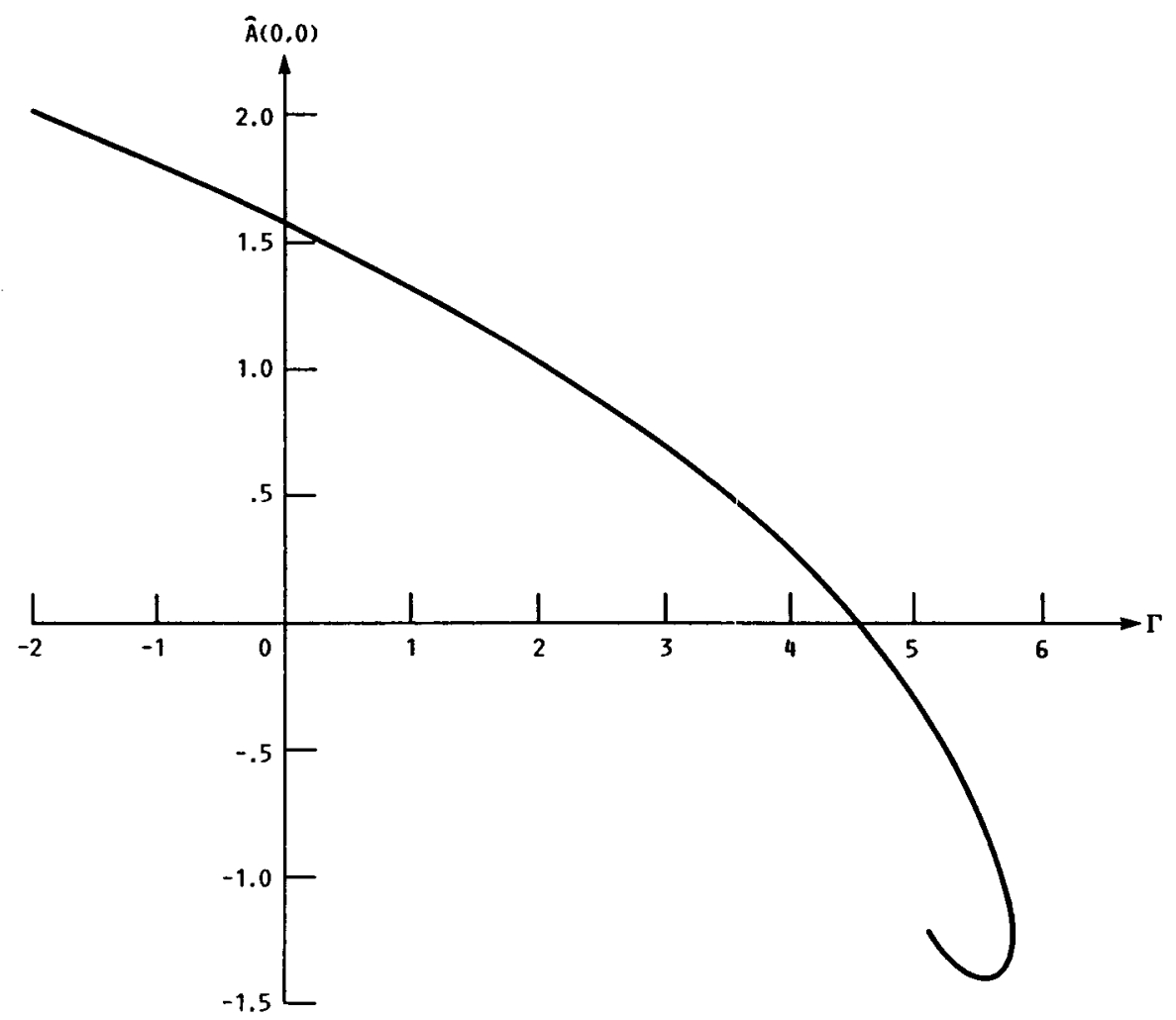

FiguRE 6. - VARIATION OF $\hat{A}(\hat{X}=0, \hat{z}=0)$ WITH $\Gamma, \lambda=2, \hat{\gamma}(\hat{z})$ DeFIMED BY $(6,1)$.

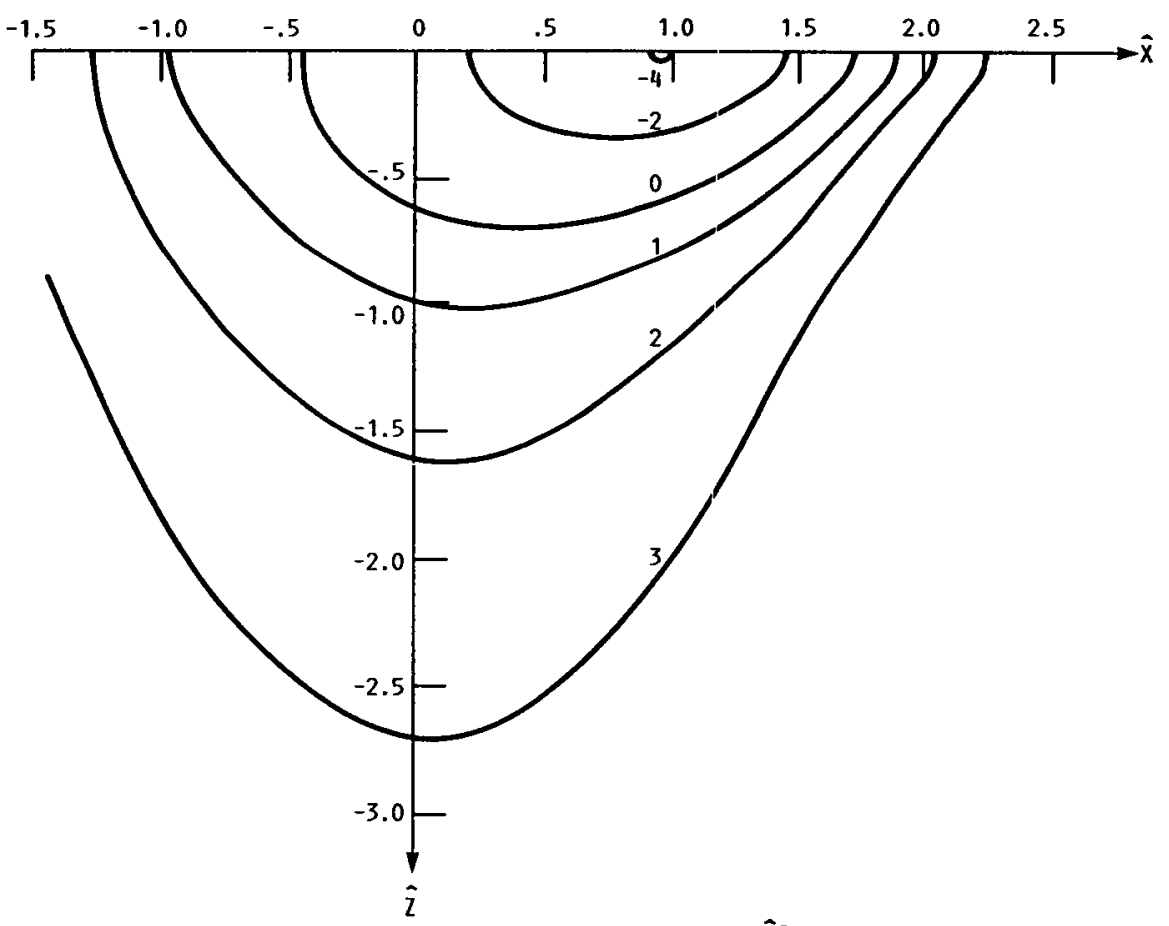

FiguRE 7. - CONTOURS FOR $\hat{A}(\hat{x}=0, \hat{z}=0), \hat{y}(z)=5.342597 e^{-\hat{z}^{2}} \lambda=2$. LOMER BRANCH SOLUTION. 


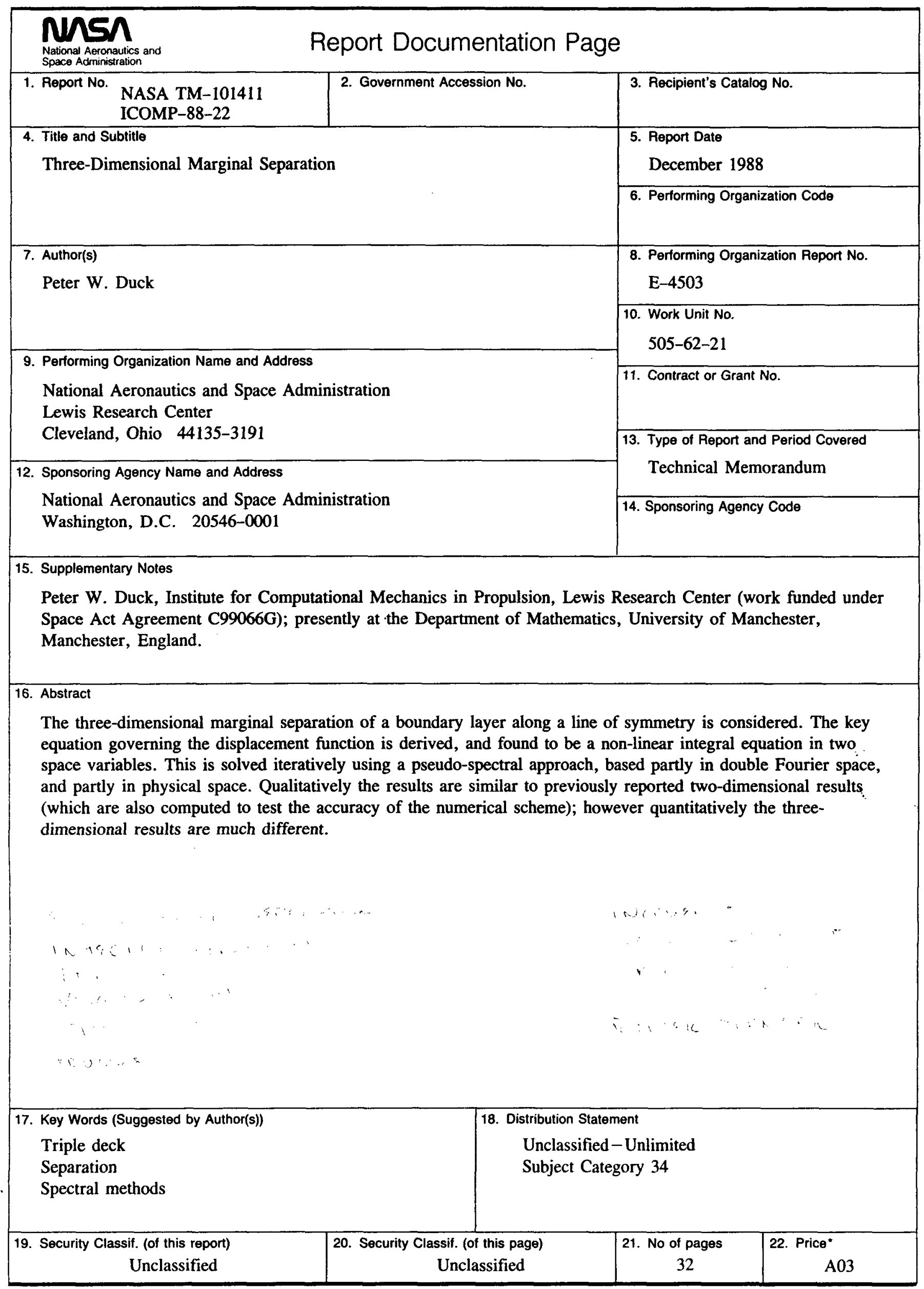

